\title{
Papers
}

\section{Domiciliary occupational therapy for patients with stroke discharged from hospital: randomised controlled trial}

\author{
Louise Gilbertson, Peter Langhorne, Andrew Walker, Ann Allen, Gordon D Murray
}

\begin{abstract}
Objective To establish if a brief programme of domiciliary occupational therapy could improve the recovery of patients with stroke discharged from hospital.

Design Single blind randomised controlled trial. Setting Two hospital sites within a UK teaching hospital.

Subjects 138 patients with stroke with a definite plan for discharge home from hospital.

Intervention Six week domiciliary occupational therapy or routine follow up.

Main outcome measures Nottingham extended activities of daily living score and "global outcome" (deterioration according to the Barthel activities of daily living index, or death).

Results By eight weeks the mean Nottingham extended activities of daily living score in the intervention group was 4.8 points $(95 \%$ confidence interval -0.5 to $10.0, \mathrm{P}=0.08)$ greater than that of the control group. Overall, 16 (24\%) intervention patients had a poor global outcome compared with $30(42 \%)$ control patients (odds ratio $0.43,0.21$ to $0.89, \mathrm{P}=0.02$ ). These patterns persisted at six months but were not statistically significant. Patients in the intervention group were more likely to report satisfaction with a range of aspects of services. Conclusion The functional outcome and satisfaction of patients with stroke can be improved by a brief occupational therapy programme carried out in the patient's home immediately after discharge. Major benefits may not, however, be sustained.
\end{abstract}

\section{Introduction}

Discharge home from hospital can be a critical stage in the rehabilitation of patients with stroke. The early recovery and new skills achieved in hospital may be difficult to transfer to the home environment. ${ }^{12}$ Poor coordination of planning of discharge, lack of access to services, psychosocial problems, and reduced quality of life are also common experiences at this time. ${ }^{13}$ Home based rehabilitation has been proposed to address these needs, and recent trials indicate that interventions for occupational therapy at home are feasible and possibly effective. ${ }^{45}$ We therefore aimed to establish a brief outreach service for occupational therapy and to evaluate whether it could improve the recovery of patients with stroke discharged home from hospital.

\section{Participants and methods}

\section{Study population}

Patients with a clinical diagnosis of stroke (excluding subarachnoid haemorrhage) who were admitted to a Glasgow royal infirmary NHS trust were eligible if they had been referred to the occupational therapy department and if a discharge date had been set. We excluded only patients for whom the service might be inappropriate (full recovery, discharge to institutional care, terminal illness), those living outside the hospital area, and those unable to take part in the trial (severe cognitive or communication problems preventing consent, completion of outcome measures, or the agreement of simple goals for recovery).

\section{Assignment}

Eligible patients were contacted by the study therapist (LG) who obtained baseline data and informed consent. Patients were told the study would compare two types of follow up; routine services (control group) or routine services plus domiciliary occupational therapy (intervention). The therapist telephoned an independent central office where baseline data were logged before allocation. Patients were randomly allocated to either intervention by a computer generated schedule stratified by sex and attendance at a day hospital contained in sequentially numbered opaque sealed envelopes. The study was approved by the local ethics committee.

\section{Interventions}

\section{Routine services}

Routine services included inpatient multidisciplinary rehabilitation, a predischarge home visit for selected patients, the provision of support services and equipment, regular multidisciplinary review at a stroke clinic, and selected patients referred to a medical day hospital.

\section{Intervention service}

The intervention service was designed to be client centred and was developed through focus group sessions with patients, carers, and local occupational therapy staff. ${ }^{3}$ From these sessions a six week domiciliary

\author{
Department of \\ Occupational \\ Therapy, Glasgow \\ Royal Infirmary, \\ Glasgow G4 0SF \\ Louise Gilbertson \\ research occupational \\ therapist \\ Ann Allen \\ helper \\ Academic Section \\ of Geriatric \\ Medicine, Glasgow \\ Royal Infirmary \\ Peter Langhorne \\ senior lecturer \\ Greater Glasgow \\ Health Board, \\ Dalian House, \\ Glasgow G3 8YU \\ Andrew Walker \\ health economist \\ Department of \\ Community Health \\ Sciences, Medical \\ Statistics Unit, \\ University of \\ Edinburgh Medical \\ School, Edinburgh \\ EH8 9AG \\ Gordon D Murray \\ professor of medical \\ statistics \\ Correspondence to: \\ P Langhorne \\ P.Langhorne@ \\ clinmed.gla.ac.uk
}

BMJ 2000;320:603-6 


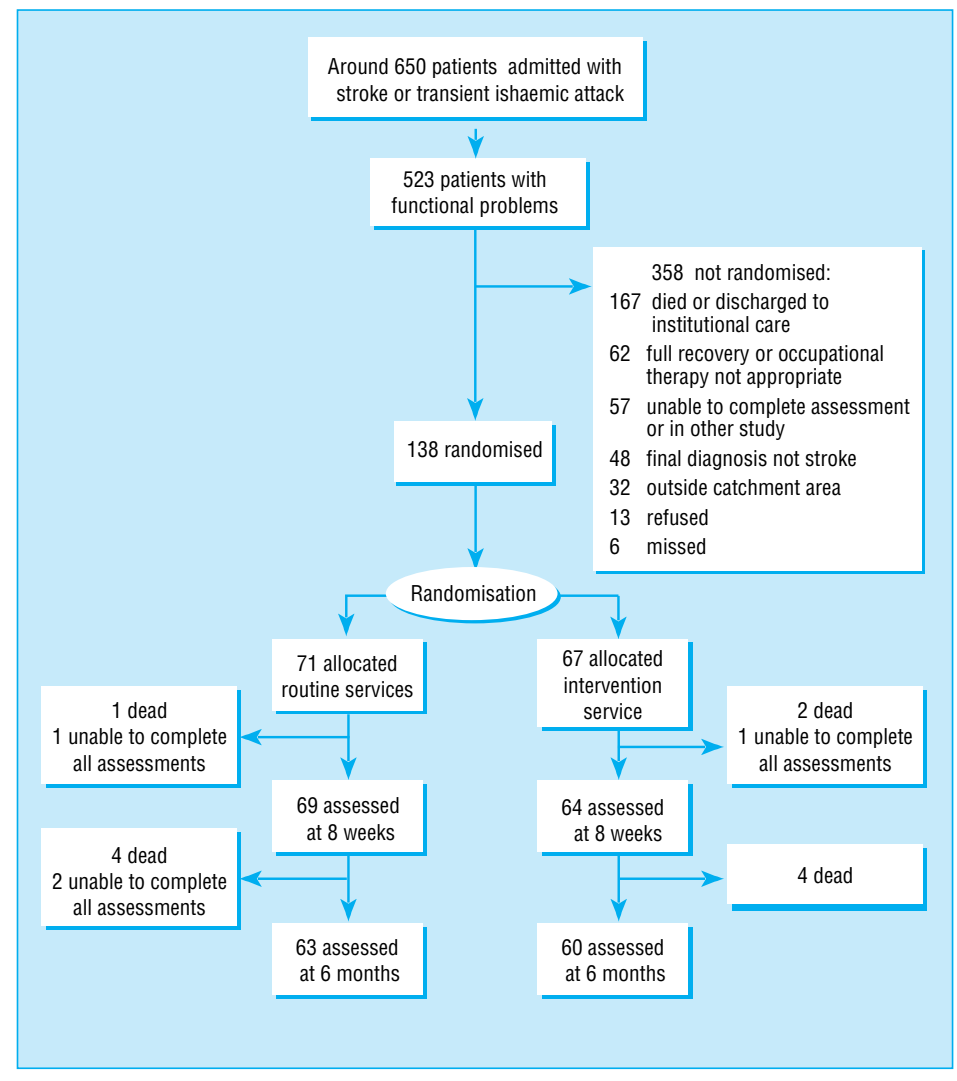

Trial profile baseline Barthel index. The total Nottingham score was well approximated by a normal distribution, and so the primary analysis was by two sample $t$ test, supported by an analysis of covariance, adjusting for baseline Barthel index and the two stratification factors of sex and referral to a day hospital. The global outcome was analysed with logistic regression, with and without adjustment for the two stratifying factors. The distribution of the Barthel index was skewed towards the upper end of the score, but normal based methods were still used as this allowed adjustment for the baseline assessment.

\section{Results}

Around 650 patients were admitted with suspected stroke or transcient ischaemic attack during an 18 month period, of whom 523 were admitted for at least three days with functional problems; we excluded 385 patients, and we randomised 67 patients to the intervention group and 71 to the control group, representing 58\% (138/237) of patients with residual stroke disability who returned to a local private address (figure).

\section{Baseline characteristics}

The intervention group tended to have more severe strokes at baseline than the control group: hemianopia, a lower Barthel index, and longer hospital stay (table 1). Three patients in the intervention group were incorrectly diagnosed with stroke (all had malignancies), and one patient in the intervention group and one in the control group were given a discharge date but never discharged. These patients remained in their allocated groups throughout this analysis. visits lasting 30-45 minutes) tailored to recovery identified by the patient such as regaining self care or domestic or leisure activities. The therapist worked with the patient to achieve these goals and also liaised with other agencies for advice, services, and equipment.

\section{Outcomes}

Baseline data were collected before randomisation, with follow up by interview after the intervention period (eight weeks) and postal outcome questionnaire at six months. The primary outcomes were the Nottingham extended activities of daily living scale and the "global outcome" of deterioration according to the Barthel activities of daily living index, or death. Secondary outcomes included the Barthel index, satisfaction with outpatient services, ${ }^{7}$ resource use (staff time, hospital readmission, provision of equipment and services), and measures of subjective health. ${ }^{3}$

We aimed to recruit a minimum of 128 patients to achieve an $80 \%$ power at $5 \%$ significance to detect a nine point difference in the Nottingham scores (66 point version) or a halving of the risk of poor global outcome. The outcome assessor (AA), who was blinded to treatment allocation, was based in a separate department from the research therapist.

\section{Statistical analyses}

All outcome data were coded, checked, and analysed on an intention to treat basis by an independent statistician. As there was a modest imbalance at baseline in severity of stroke a post hoc decision was made to explore the effects of adjusting the analysis for the

\section{Functional outcomes}

The unadjusted analysis at eight weeks (table 2) showed that the intervention group had a mean

Table 1 Patient characteristics at randomisation. Values are numbers (percentages) unless stated otherwise

\begin{tabular}{lcc} 
Characteristics & $\begin{array}{c}\text { Intervention } \\
\text { group (n=67) }\end{array}$ & $\begin{array}{c}\text { Control } \\
\text { group } \\
\mathbf{( n = 7 1 )}\end{array}$ \\
\hline Patient & $71(28-89)$ & $71(31-89)$ \\
\hline Age (years; interquartile range) & $29(43)$ & $31(44)$ \\
\hline Male & $31(46)$ & $24(34)$ \\
\hline Living alone & $62(93)$ & $66(93)$ \\
\hline Rankin score before stroke: & $5(7)$ & $5(7)$ \\
\hline $0-2$ & & \\
\hline $3-4$ & $33(49)$ & $38(53)$ \\
\hline Stroke & $60(90)$ & $60(84)$ \\
\hline Laterality: right hemisphere & $19(28)$ & $14(20)$ \\
\hline Hemiparesis & $13(19)$ & $16(22)$ \\
\hline Hemisensory loss & $16(24)$ & $7(10)$ \\
\hline Visual or spatial inattention & $22(33)$ & $16(22)$ \\
\hline Hemianopia & $9(13)$ & $4(6)$ \\
\hline Dysphasia & $13(20)$ & $19(27)$ \\
\hline Brain stem or cerebellar signs & $14(21)$ & $17(24)$ \\
\hline Cognitive impairment & $5(7)$ & $5(7)$ \\
\hline Anxiety & $16(24)$ & \\
\hline Depression & $21(30)$ \\
\hline Other & $31(17-57)$ & $23(13-66)$ \\
\hline Median duration (days) since symptom onset & & \\
\hline interquartile range) & & \\
\hline Median Barthel index score (interquartile range) & $17(15-18)$ & $18(16-19)$ \\
\hline Referral to medical day hospital & $16(14)$ \\
\hline
\end{tabular}


Nottingham score 4.8 points higher than that of the control group ( $95 \%$ confidence interval -0.5 to 10.0 , $\mathrm{P}=0.08)$. In the corresponding adjusted analysis the mean difference was 5.7 points (1.2 to $10.3, \mathrm{P}=0.02$ ). Significantly fewer patients in the intervention group experienced a poor global outcome (odds ratio 0.43, 0.21 to $0.89, \mathrm{P}=0.02$ ). A similar pattern was seen with the Barthel index scores (table 2).

At six months the results for primary outcomes were no longer statistically significant (table 2). More patients in the intervention than control group were, however, likely to have improved and the change in Barthel index scores in these patients was significantly better than in the controls.

\section{Patient satisfaction}

Overall, 44 patients in the intervention group and 43 in the control group returned a questionnaire about satisfaction with service delivery at home. ${ }^{7}$ Patients in the intervention group were more likely to report satisfaction across all 12 questions (summary odds ratio for agreement with statements $1.8,1.4$ to 2.4 ). In particular they were significantly more likely to agree that "things were well prepared for returning home" and that they "knew who to contact with problems relating to my stroke."

\section{Resource use}

The groups were evenly matched at the six months' follow up for place of residence, readmissions to hospital, additional services and equipment provided, and costs incurred by patients and carers. ${ }^{3}$ Staff costs (including travel) accounted for 85\%-90\% of all expenditure. We estimate that one whole time therapist could manage 80-90 patients per year at a cost of about $£ 300-£ 320$ per patient and prevent 10 poor outcomes (deterioration in function) after discharge home-that is, costing about $£ 2500$ per poor outcome avoided.

\section{Unblinding}

The outcome assessor was asked to guess the allocation of the last 46 patients followed up, and she guessed correctly in 32 cases $(69 \%, 56 \%$ to $83 \%)$. The commonest reason was knowing whether the patient had attempted an activities of daily living activity (in particular bathing), which should have been addressed in the occupational therapy programme.

\section{Discussion}

\section{Methodological issues}

Our trial shows that patients with stroke who have received multidisciplinary rehabilitation in hospital incorporating discharge planning and multidisciplinary follow up can still benefit from a short outreach programme for occupational therapy. The initial statistically significant benefits were diminished at the six month follow up, which could reflect the method of follow up (postal versus interview) or a transient effect of the rehabilitation input. It is possible that the early benefits were maintained at six months as the wide confidence intervals do not exclude this possibility. We have tried to ensure a rigorous but pragmatic evaluation of a new service using a rigorous randomisation procedure and independent intention to treat
Table 2 Main functional outcome data. Values are medians (interquartile ranges) unless stated otherwise

\begin{tabular}{cccccc} 
& \multicolumn{2}{c}{ Descriptive } & statistics & & \multicolumn{2}{c}{ Unadjusted analysis } \\
\cline { 2 - 3 } \cline { 6 - 7 } Outcome & $\begin{array}{c}\text { Intervention } \\
(n=67)\end{array}$ & $\begin{array}{c}\text { Control } \\
(n=71)\end{array}$ & & $\begin{array}{c}\text { Difference } \\
(95 \% ~ C I)\end{array}$ & $\begin{array}{c}P \\
\text { value }\end{array}$ \\
\hline
\end{tabular}

8 weeks

\begin{tabular}{lllll}
\hline No of deaths & 2 & 1 & - & - \\
\hline No unable to complete all assessments & 1 & 1 & - & -
\end{tabular}

Nottingham extended activities of daily living score:

\begin{tabular}{|c|c|c|c|c|}
\hline Mobility & $7(4-12)$ & $6(2-10)$ & $1(-0.3$ to 3.1$)$ & 0.11 \\
\hline Domestic & $15(9-23)$ & $11(4-20)$ & $4(0.0$ to 5.7$)$ & 0.05 \\
\hline Leisure & $7(3-9)$ & $6(3-9)$ & $1(-0.8$ to 1.8$)$ & 0.44 \\
\hline Total $^{*}$ & $27(19-43)$ & $23(11-35)$ & $4(-0.5$ to 10.0$)$ & 0.08 \\
\hline Barthel activities of daily living index & $18(16-20)$ & $17(14-19)$ & $1(0.0$ to 2.3$)$ & 0.06 \\
\hline $\begin{array}{l}\text { Change in Barthel index score since } \\
\text { randomisation }\end{array}$ & $1(0-2)$ & $0(-3-1)$ & $1(0.7$ to 2.7$)$ & 0.001 \\
\hline \multicolumn{5}{|l|}{ Global outcome: } \\
\hline $\begin{array}{l}\text { No (\%) with improved activities of } \\
\text { daily living }\end{array}$ & $38(57)$ & $25(35)$ & - & 0.03 \\
\hline No (\%) with no change & $13(19)$ & $16(24)$ & - & - \\
\hline $\begin{array}{l}\text { No (\%) with worse activities of daily } \\
\text { living or who had died }\end{array}$ & $16(24)$ & $30(42)$ & - & - \\
\hline Odds of a poor global outcome ${ }^{*}$ & - & - & $0.43(0.21$ to 0.89$)$ & 0.02 \\
\hline \multicolumn{5}{|l|}{6 months } \\
\hline No of deaths & 6 & 5 & - & - \\
\hline No unable to complete all assessments & 1 & 3 & - & - \\
\hline \multicolumn{5}{|c|}{ Nottingham extended activities of daily living score: } \\
\hline Mobility & $8(4-12)$ & $6(3-11)$ & $2(-1.0$ to 2.5$)$ & 0.38 \\
\hline Domestic & $14(6-21)$ & $12(5-19)$ & $2(-1.7$ to 4.5$)$ & 0.36 \\
\hline Leisure & $6(3-10)$ & $6(3-9)$ & $0(-1.6$ to 1.3$)$ & 0.82 \\
\hline Total $^{*}$ & $28(15-38)$ & $21(14-38)$ & $7(-3.6$ to 7.8$)$ & 0.48 \\
\hline Barthel activities of daily living index & $17(15-19)$ & $17(13-18)$ & $0(-0.6$ to 2.4$)$ & 0.25 \\
\hline Change in Barthel index since randomisation & $0(-2-2)$ & $-1(-3-0)$ & $-1(0.1$ to 2.9$)$ & 0.04 \\
\hline \multicolumn{5}{|l|}{ Global outcome: } \\
\hline $\begin{array}{l}\text { No (\%) with improved activities of } \\
\text { daily living }\end{array}$ & $27(42)$ & $15(22)$ & - & 0.04 \\
\hline No (\%) with no change & $6(9)$ & $11(16)$ & - & - \\
\hline $\begin{array}{l}\text { No (\%) with worse activities of } \\
\text { daily living or who had died }\end{array}$ & $32(49)$ & $42(62)$ & - & - \\
\hline Odds of a poor global outcome ${ }^{\star}$ & - & - & $0.60(0.30$ to 1.20$)$ & 0.15 \\
\hline
\end{tabular}

*Primary outcomes.

analysis. We do, however, acknowledge the difficulty in blinding rehabilitation trials. Unblinding of the outcome assessor may not have biased recording of outcomes since the main reason she guessed treatment allocation was differences in components of the outcome measures. The final (six month) outcomes were reported by postal questionnaire and so were not prone to observer unblinding. Patients' responses may have been influenced by their knowledge of their allocated group, ${ }^{7}$ but it is difficult to exclude this possibility in a pragmatic trial with informed consent.

\section{Comparison with other studies}

The main weakness of our study is its comparatively small size, which meant it was prone to baseline differences between patient groups and had limited power to detect a modest effect on functional outcomes. Our power calculations were based on previous studies, which in comparison with our trial provided a comparatively prolonged therapy input to the intervention group and little input to controls. ${ }^{2}{ }^{45}$ We were therefore probably trying to detect smaller differences between intervention and control patients than was originally anticipated. Interestingly, a recent trial showing a positive impact of domiciliary occupational therapy also had a control group that received minimal input. ${ }^{8}$ The apparently transient benefit of our 
What is already known on this topic

Patients with stroke returning home from hospital often encounter unanticipated disability and difficulties in adapting to the home environment

No intervention has been shown to alleviate these problems

\section{What this study adds}

A brief programme of domiciliary occupational therapy can enhance recovery and reduce the risk of deterioration in patients with stroke returning home

Rehabilitation should be extended beyond discharge from hospital

intervention has been observed previously and used to justify a more prolonged therapy input. ${ }^{45}$ Reducing the longer term impact of stroke remains a major challenge.

\section{Implications}

Our results lend support to the principle of extending routine stroke rehabilitation from the inpatient period to postdischarge period. Our resource analysis shows that the service costs are significant but that one therapist could manage 80-100 patients per year and prevent about 10 deteriorating in function after discharge home. We did not attempt to reduce hospital stay, but two recent British trials of early hospital discharge with a domiciliary multidisciplinary rehabilitation have shown a shortening of the period of inpatient care with no apparent adverse effect on patient outcomes. ${ }^{10} 11$ If confirmed, this potentially offers a way of improving postdischarge rehabilitation without incurring major additional service costs.

We thank the patients and carers who contributed to the study.

Contributors: LG planned and conducted the trial and drafted the final report. PL planned and supervised the trial; he will act as guarantor for the paper. AW provided health economic input and analysis. AA contributed to the design and conduct of the trial. GDM provided independent data analysis and statistical advice. All authors contributed to the redrafting and approval of the final report.

Funding: Chest Heart and Stroke Scotland provided the funding for this study. Additional support came from Glasgow Royal Infirmary NHS Trust and the chief scientists office, Scottish Office, which funded a research training fellowship for LG.

Competing interests: None declared.

Forster A, Young J. Stroke rehabilitation: can we do better? BMJ 1992:305:1446-7.

2 Corr S, Bayer A. Occupational therapy for stroke patients after hospital discharge. Clin Rehab 1995;9:291-6.

3 Gilbertson L. A randomised controlled trial of home based occupational therapy for stroke patients. MSc thesis. University of Glasgow, 1998.

Drummond AER, Walker MF. A randomised controlled trial of leisure rehabilitation. Clin Rehab 1995;9:283-90.

5 Logan PA, Gladman JRF, Lincoln NB. A randomised controlled trial of enhanced social service occupational therapy for stroke patients. Clin Rehab 1997;11:107-13.

6 Wade DT. Measurement in neurological rehabilitation. Oxford: Oxford University Press, 1992.

7 Dennis MS, O'Rourke S, Slattery J, Staniforth T, Warlow C. Evaluation of a stroke family care worker: results of a randomised controlled trial. BMJ 1997;314:1071-7.

8 Walker MF, Gladman JRF, Lincoln N, Siemonsma P, Whiteley T. Occupational therapy for stroke patients not admitted to hospital: a randomised controlled trial. Lancet 1999;354:278-80

9 Gladman J, Barer D, Langhorne P. Specialist rehabilitation after stroke. Effective in the short term, but more work needed in the long term. BMJ 1996;312:1623-4.

10 Rudd AG, Wolfe CDA, Filling K, Beech R. Randomised controlled trial to evaluate early discharge scheme for patients with stroke. BMJ 1997:315:1039-44.

11 Rodgers H, Soutter J, Kaiser W, Pearson P, Dobson R, Skilbeck C, et al. Early supported hospital discharge following acute stroke: pilot study results. Clin Rehab 1997;11:280-7.

(Accepted 13 December 1999)

\title{
Six year survey of screening for Down's syndrome by maternal age and mid-trimester ultrasound scans
}

\author{
David T Howe, Robert Gornall, Diana Wellesley, Tracy Boyle, John Barber
}

\section{Editorial by \\ Raeburn \\ Wessex Maternal and Fetal Medicine Unit, Princess Anne Hospital, Southampton SO16 5YA \\ David T Howe consultant in fetomaternal medicine Robert Gornall specialist registrar in obstetrics and gynaecology continued over}

BMJ 2000;320:606-10

\author{
Abstract \\ Objective To assess the effectiveness of antenatal \\ screening for Down's syndrome by maternal age and \\ routine mid-pregnancy ultrasound scanning. \\ Design Retrospective six year survey. \\ Setting Maternity units of a district general hospital. \\ Subjects Pregnant women booked for delivery in \\ hospital between 1 January 1993 and 31 December \\ 1998. \\ Main outcome measures All cases of Down's \\ syndrome occurring in district identified from \\ regional congenital anomaly register and cytogenetic \\ laboratory records. Women's case notes were \\ examined to identify indication for karyotyping, \\ gestation at diagnosis, and outcome of pregnancy. \\ Results 31259 deliveries occurred during study \\ period, and 57 cases of Down's syndrome were
}

identified, four in failed pregnancies and 53 in ongoing pregnancies or in neonates. The analysis was confined to ongoing pregnancies or liveborn children. Invasive antenatal tests were performed in $6.6 \%$ (2053/31 259), and 68\% (95\% confidence interval $56 \%$ to $80 \%$ ) of cases of Down's syndrome were detected antenatally, giving a positive predictive value of $1.8 \%$. There were 17 undetected cases, and in seven of these the women had declined an offer of invasive testing. In women aged less than 35 years the detection rate was $53 \%(30 \%$ to $76 \%)$. Most of the cases detected in younger women followed identification of ultrasound anomalies.

Conclusions The overall detection rate was considerably higher than assumed in demonstration projects for serum screening. As a result, the benefits of serum screening are much less than supposed. Before any new methods to identify Down's syndrome 